\title{
Physical Scientists
}

National Cancer Institute

\section{Source}

National Cancer Institute. Physical Scientists. NCI Thesaurus. Code C122497.

The group of science professions that include astronomers, physicists, atmospheric and space scientists, chemists, materials scientists, environmental scientists and specialists, geoscientists, and hydrologists. 